University of Nebraska - Lincoln

DigitalCommons@University of Nebraska - Lincoln

$1-28-2008$

\title{
Proteresis in Co:CoO core-shell nanoclusters
}

\author{
X.-H. Wei \\ University of Nebraska-Lincoln \\ Ralph Skomski \\ University of Nebraska-Lincoln, rskomski2@unl.edu \\ Zhiguang Sun \\ University of Nebraska-Lincoln, zsun3@unl.edu \\ David J. Sellmyer \\ University of Nebraska-Lincoln, dsellmyer@unl.edu
}

Follow this and additional works at: https://digitalcommons.unl.edu/physicsskomski

Part of the Physics Commons

Wei, X.-H.; Skomski, Ralph; Sun, Zhiguang; and Sellmyer, David J., "Proteresis in Co:CoO core-shell nanoclusters" (2008). Ralph Skomski Publications. 48.

https://digitalcommons.unl.edu/physicsskomski/48

This Article is brought to you for free and open access by the Research Papers in Physics and Astronomy at DigitalCommons@University of Nebraska - Lincoln. It has been accepted for inclusion in Ralph Skomski Publications by an authorized administrator of DigitalCommons@University of Nebraska - Lincoln. 


\title{
Proteresis in Co:CoO core-shell nanoclusters
}

\author{
X.-H. Wei, ${ }^{\text {a) }}$ R. Skomski, Z.-G. Sun, and D. J. Sellmyer \\ Department of Physics and Astronomy and Nebraska Center for Materials and Nanoscience, \\ University of Nebraska, Lincoln, Nebraska 68588, USA
}

(Presented on 8 November 2007; received 12 September 2007; accepted 2 October 2007; published online 28 January 2008)

\begin{abstract}
The magnetism of ultrasmall $\mathrm{Co}: \mathrm{CoO}$ core-shell nanoclusters is investigated. The structures, produced by cluster-beam deposition, have Co core sizes ranging from 1 to $7 \mathrm{~nm}$ and a CoO shell thickness of about $3 \mathrm{~nm}$. Hysteresis loops as well as zero-field-cooled and field-cooled magnetization curves have been measured and a striking feature of the nanostructures is the existence of proteretic (clockwise) rather than hysteretic loops in the core-size range from 3 to $4 \mathrm{~nm}$. The proteretic behavior and its particle-size dependence reflect a subtle interplay between various anisotropies and exchange interactions in the $\mathrm{Co}$ and $\mathrm{CoO}$ phases and at the Co-CoO interface. (C) 2008 American Institute of Physics. [DOI: 10.1063/1.2829239]
\end{abstract}

\section{INTRODUCTION}

Since the discovery of unidirectional anisotropy more than 50 years ago, ${ }^{1}$ the $\mathrm{Co}: \mathrm{CoO}$ system has attracted continuing attention. A poorly understood aspect is the occurrence of inverted hysteresis loops or proteresis. Such loops, found in some nanostructures with competing magnetic interactions, ${ }^{2}$ are cycled clockwise, in contrast to ordinary or anticlockwise hysteresis loops. This is different from loops shifted horizontally or vertically due to exchange bias, and from constricted hysteresis loops, also known as waspshaped or butterfly loops. Proteretic loops are encountered, for example, in pharmacodynamics, where one plots the effect of a drug as a function of the drug concentration. ${ }^{3}$ Similar effects occur in transport systems such as superconductors. ${ }^{4}$ In magnetism, they are counterintuitive, because the clockwise character of the loops indicates the production rather than dissipation of magnetic energy. The reason for the occurrence of magnetic proteresis is the involvement of competing interactions, especially exchange.

Inverted loops were originally obtained and qualitatively explained in granular thin films. ${ }^{2}$ Recent advances in cluster processing ${ }^{5,6}$ have provided the basis for structuring nanoparticles with well-defined and narrow size ranges. The phenomena considered in this paper share distant relationships with the exchange coupling in hard-soft nanocomposites ${ }^{7-9}$ and with exchange bias. ${ }^{1,10}$ The former phenomenon exhibits some similarities, such as a size-dependent exchange coupling to a soft phase, but it does not involve competing exchange interactions. The latter has the same physical origin, namely, the interplay between ferromagnetic and antiferromagnetic exchanges, but maps these interactions on a simple exchange field.

Here we produce $\mathrm{Co}: \mathrm{CoO}$ core-shell clusters and investigate the magnetism of the nanostructures, with particular reference to magnetic proteresis in surface-modified nanoclusters, which are an emerging research field with potential

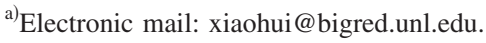

applications in areas such as ultrahigh-density magnetic recording, magnetoresistive sensors, contrasting agents in medicine, and catalysis. ${ }^{11-13}$

\section{SAMPLE PREPARATION AND CHARACTERIZATION}

Nearly monodisperse Co nanoclusters having sizes from 1 to $7 \mathrm{~nm}$ were produced in a cluster-beam deposition system, as described, for example, in Ref. 14. The core-shell clusters were then produced by partial oxidation in an oxygen atmosphere of fixed flow rate before they were deposited into a silica matrix to form pseudomultilayers. To avoid intercluster interactions, we have focused on dilute pseudomultilayers with a nominal cluster-layer thickness of $1 \AA$ in a silica matrix of thickness $15 \mathrm{~nm}$. However, for comparison, we have also produced dense-packed series. Figure 1 shows the schematic structure.

The formation of uniform $\mathrm{Co}$ : $\mathrm{CoO}$ core-shell clusters of various sizes is confirmed by transmission electron microscopy (TEM) (Fig. 2). While the core-shell particles are too small for phase analysis using $\mathrm{X}$-ray diffraction, the oxidation of $\mathrm{Co}$ is known to yield $\mathrm{CoO},{ }^{15,16}$ and the corresponding core-shell structure is consistent with our magnetic measurements. In contrast to $\mathrm{CoO}$, other oxides such as $\mathrm{Co}_{2} \mathrm{O}_{3}$ and $\mathrm{Co}_{3} \mathrm{O}_{4}$ require high oxygen flow rates, as explained in Ref. 17. With relatively low oxygen flow rate other oxide is not expected in our samples. The Co core is in the fcc phase, as concluded from the x-ray diffraction examination of bare and

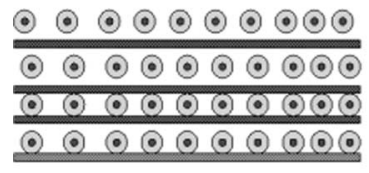

(a)

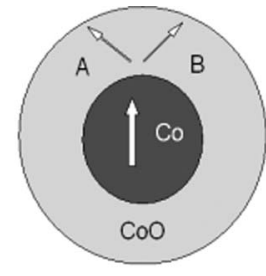

(b)
FIG. 1. Geometry of CoO:Co core-shell nanostructures: (a) pseudomultilayer and (b) core-shell particle with $A$ and $B$ showing sublattice magnetizations in $\mathrm{CoO}$. 


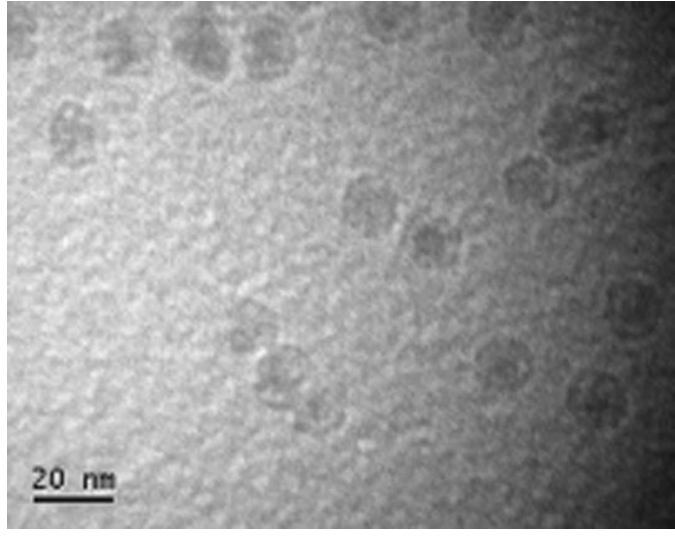

FIG. 2. TEM image of core-shell clusters having a $\mathrm{CoO}$ shell thickness of $3 \mathrm{~nm}$ and Co core diameters of $7 \mathrm{~nm}$.

unoxidized clusters. Note that variable oxygen flow rates could lead to different oxidation degrees and shell thicknesses, but in the present work, oxygen flow rate was fixed to ensure that the structures have a common shell thickness of about $3 \mathrm{~nm}$. High resolution TEM analysis of clusters made with the same method in the past ${ }^{17}$ suggests that the $\mathrm{CoO}$ shell consists of nanocrystallites in the cubic (NaCl-type) crystal structure.

\section{MAGNETIC PROPERTIES}

The magnetic properties of the clusters were measured using the superconducting quantum interference device in the temperature range from 5 to $300 \mathrm{~K} . M(H)$ loops and magnetizations after both zero-field cooling and field cooling were measured. We have also performed atomic force microscopy and magnetic force microscopy imaging on the silica-embedded core-shell structures, but the resolution of the images (not shown in this paper) is insufficient to study individual core-shell clusters.

The zero-field-cooled (ZFC) and field-cooled (FC) magnetizations were obtained in a standard way, and the blocking temperature $T_{p}$ is estimated from the peak (or maximum) of the ZFC curve. Figure 3(a) shows the FC and ZFC curves for $7 \mathrm{~nm}$ core-shell clusters and Fig. 3(b) gives the dependence of the blocking temperature on the core diameter $D$. The experimental data are reasonably well reproduced by a phenomenological power law, $T_{p} \sim D^{2} / V_{s}^{3 / 2}$, solid line in (b), where $V_{s}$ is the volume of the $\mathrm{CoO}$ shell. The quadratic dependence on $D$ indicates that the core-shell interface plays an important role. This does not necessarily imply substantial interface anisotropy. A more likely explanation is that the
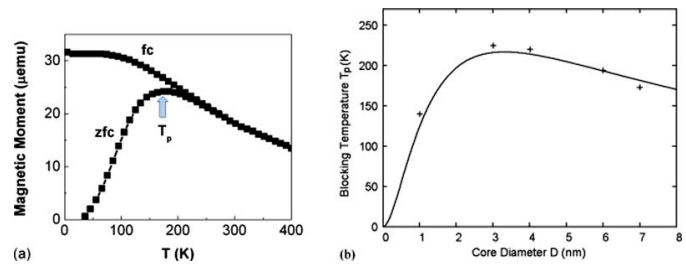

FIG. 3. (Color online) Blocking behavior of the core-shell structures: (a) ZFC and FC magnetization curves for $7 \mathrm{~nm}$ clusters, and (b) blocking temperature $T_{p}$ as a function of core diameter. The solid curve in (b) is a model prediction.
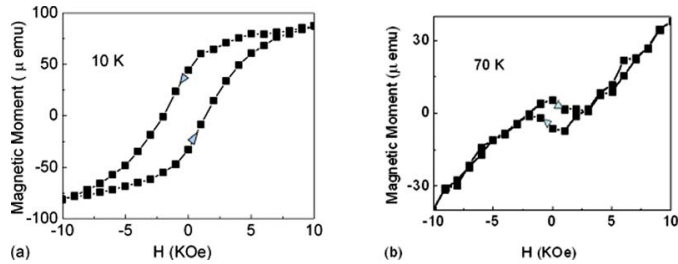

FIG. 4. (Color online) Magnetization curves for (a) $D=1 \mathrm{~nm}$ (hysteresis) and (b) $D=3 \mathrm{~nm}$ (proteresis).

relatively soft $\mathrm{Co}$ couples to adjacent $\mathrm{CoO}$ regions (crystallites) and that the $\mathrm{CoO}$ volume involved in this coupling is proportional to the interface area.

The pronounced decrease of $T_{p}$ with increasing size may have two reasons. First, the $\mathrm{CoO}$ is nanocrystalline (x-ray amorphous) rather than single crystalline, which leads to some random-anisotropy averaging in the $\mathrm{CoO}$ scaling as $1 / V_{s}^{1 / 2}$. Note that random-anisotropy averaging is well known in ferromagnets ${ }^{18}$ but also occurs in antiferromagnets, where it becomes visible near spin-flop transitions ${ }^{19}$ or, in the present, by exchange coupling to a ferromagnet. Second, the coupling due to the $\mathrm{Co}$ core is strongest for neighboring $\mathrm{CoO}$ grains, so that more distant grains are effectively decoupled. A more detailed discussion of the $T_{p}(D)$ dependence is difficult, because $V_{s}$ and $D$ are strongly coupled and the shell thickness $D$ is difficult to tune. For example, small $D$ leads to the formation of $\mathrm{CoO}$ islands on the Co surface, which affects the above-mentioned random-anisotropy argument.

Depending on the core diameter, the $M(H)$ loops are clockwise (proteretic) or anticlockwise (hysteretic). Proteretic loops are observed in a certain core-size window, from about $3 \mathrm{~nm}$ to about $4 \mathrm{~nm}$. Figure 4 shows typical loops for different core sizes.

The densely packed samples exhibit vertical loop shifts, in addition to the more common horizontal exchange bias. This could be understood by the strengthening of the $\mathrm{CoO}$ shell due to contact among the core-shell clusters, thus enhancing the magnitude of exchange coupling. Combination of vertical and horizontal shifts produces a "rotating" behavior with increasing temperature. Both shifts have the same origin and can be considered as inner loops from a micromagnetic point of view, but they complicate the quantitative understanding of the $M(H)$ loops. Shown in Fig. 5 are the rotating loops and the temperature dependence of vertical shift.

\section{DISCUSSION AND CONCLUSIONS}

Clockwise hysteresis or proteresis, as shown in Fig. 4(b), is much less common than ordinary hysteresis, which is char-
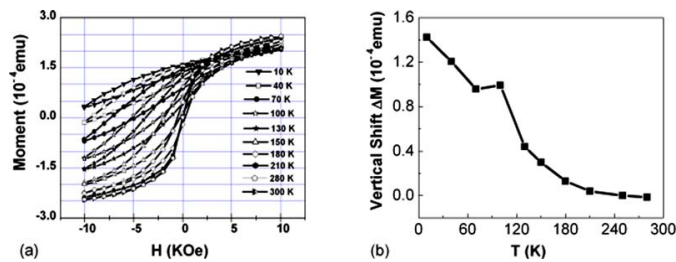

FIG. 5. (Color online) Temperature dependence of hysteresis loops (a) and vertical shifts (b) of compact films. 
acterized by counterclockwise $M(H)$ loops. Since clockwise or inverted loops produce rather than dissipate magnetic energy, the phenomenon is somewhat counterintuitive, indicating that the magnetic energy is outweighed by other energy contributions, such as interatomic exchange. A semiquantitive explanation of clockwise hysteresis loops in granular ferromagnetic/antiferromagnetic (FM/AFM) composites has been given by O'Shea and Al-Sharif. ${ }^{2}$ The essence of the explanation is that the external or Zeeman field changes the sublattice magnetizations $A$ and $B$ in Fig. 1(b). The Co magnetization wants to follow the Zeeman field, too, but it is also coupled to the $\mathrm{CoO}$ sublattices, and the switching of the $\mathrm{CoO}$ magnetizations $A$ and $B$ may actually overcompensate the Zeeman interaction and switch the Co magnetization in the opposite direction. In more detail, the explanation involves three types of exchange, namely, (i) the predominantly AFM intra- and intersublattice exchange in the $\mathrm{CoO}$, (ii) the ferromagnetic exchange in the $\mathrm{Co}$, and (iii) the $\mathrm{Co} / \mathrm{CoO}$ interfacial exchange, which competes with the AFM exchange of the $\mathrm{CoO}$. The magnetocrystalline magnetic energy required for proteresis is largely provided by the $\mathrm{CoO}$ phase.

Of course, this explanation is rather general and applies to broad range of FM/AFM structures. ${ }^{2,20}$ What is the reason for the window $(2 \mathrm{~nm}<D<5 \mathrm{~nm})$ where the effect occurs in the $\mathrm{Co}: \mathrm{CoO}$ core-shell structures? To explain the size dependence, we first consider soft ferromagnetic regions (inclusions) in a hard but ferromagnetic matrix. The hard and soft regions are strongly exchange coupled when the dimension of the soft region does not exceed a few nanometers. ${ }^{7,21} \mathrm{Re}-$ placing the anisotropic ferromagnetic matrix by an anisotropic antiferromagnetic matrix complicates the micromagnetism of the system but leaves the involved length scales unchanged. In both cases, very small length scales lead to nearly complete effective exchange coupling, and the magnet behaves like a single hysteretic unit. Only on a length scale of a few nanometers, where the involved interactions are of comparable strength, does one observe proteresis.

In summary, proteresis (clockwise hysteresis) is observed in $\mathrm{Co}: \mathrm{CoO}$ core-shell particles. The phenomenon is limited to Co core sizes from about 3 to $4 \mathrm{~nm}$-cores smaller than $3 \mathrm{~nm}$ and larger than $4 \mathrm{~nm}$ lead to ordinary hysteresis loops. Proteresis reflects a complicated interplay between ferromagnetic and antiferromagnetic exchange interactions and anisotropies; it is a true nanoscale phenomenon, not occurring in atomic-scale and macroscopic magnets. Future research is necessary to quantify this intriguing size dependence.

\section{ACKNOWLEDGMENTS}

This research is supported by NSF MRSEC under Grant No. 0213808 and NCMN.

${ }^{1}$ W. H. Meiklejohn and C. P. Bean, Phys. Rev. 102, 1413 (1956).

${ }^{2}$ M. J. O'Shea and A. L. Al-Sharif, J. Appl. Phys. 75, 6673 (1994).

${ }^{3}$ P. Girard and J.-P. Boissel, J. Pharmacokinetics and Pharmacodynamics 17, 401 (1989)

${ }^{4}$ A. V. Samoilov, M. Konczykowski, N.-C. Yeh, S. Berry, and C. C. Tsuei, Phys. Rev. Lett. 75, 4118 (1995).

${ }^{5}$ A. N. Dobrynin, D. N. Ievlev, C. Hendrich, K. Temst, P. Lievens, U. Hörmann, J. Verbeeck, G. Van Tendeloo, and A. Vantomme, Phys. Rev. B 73, 245416 (2006).

${ }^{6}$ Y. F. Xu, M. L. Yan, and D. J. Sellmyer, in Advanced Magnetic Nanostructures, edited by D. J. Sellmyer and R. Skomski (Springer, Berlin, 2006), Chap. 8, p. 207.

${ }^{7}$ R. Skomski and J. M. D. Coey, Phys. Rev. B 48, 15812 (1993).

${ }^{8}$ E. E. Fullerton, J. S. Jiang, C. H. Sowers, J. E. Pearson, and S. D. Bader, Appl. Phys. Lett. 72, 380 (1998).

${ }^{9}$ J. P. Liu, C. P. Luo, Y. Liu, and D. J. Sellmyer, Appl. Phys. Lett. 72, 483 (1998).

${ }^{10}$ B. H. Miller and E. Dan Dahlberg, Appl. Phys. Lett. 69, 3932 (1996).

${ }^{11}$ J. Antony, Y. Qiang, D. R. Baer, and C. Wang, J. Nanosci. Nanotechnol. 6, 568 (2006).

${ }^{12}$ T. Munoz, J. A. De Toro, P. S. Normile, J. P. Andres, J. A. Gonzalez, P. Muniz, A. J. Barbero, and J. M. Riveriro, J. Appl. Phys. 101, 09E504 (2007).

${ }^{13}$ J. Anthony, Y. Qiang, D. Baer, and C. Wang, J. Nanosci. Nanotechnol. 6, 568 (2006)

${ }^{14}$ Y. Qiang, R. F. Sabiryanov, S. S. Jaswal, Y. Liu, H. Haberland, and D. J. Sellmyer, Phys. Rev. B 66, 064404 (2002).

${ }^{15}$ Y. J. Tang, D. J. Smith, B. L. Zink, F. Hellman, and A. E. Berkowitz, Phys. Rev. B 67, 054408 (2003).

${ }^{16}$ D.-L. Peng, K. Sumiyama, and T. Hihara, Jpn. J. Appl. Phys., Part 139, 66 (2000).

${ }^{17}$ J. A. De Toro, J. P. Andres, J. A. Gonzale, P. Muniz, T. Munoz, P. S. Normile, and J. M. Riveiro, Phys. Rev. B 73, 094449 (2006).

${ }^{18}$ E. Callen, Y. J. Liu, and J. R. Cullen, Phys. Rev. B 16, 263 (1977).

${ }^{19}$ J. Richter and R. Skomski, Phys. Status Solidi B 153, 711 (1989).

${ }^{20}$ Y. Z. Wu, G. S. Dong, and X. F. Jin, Phys. Rev. B 64, 214406 (2001).

${ }^{21}$ R. Skomski, J.-P. Liu, and D. J. Sellmyer, Phys. Rev. B 60, 7359 (1999). 